\title{
Fibra de coco y cáscara de plátano como alternativa para la elaboración de material biodegradable
}

\section{Coconut fiber and plantain banana peel as an alternative for the production of biodegradable material}

\author{
Cubilla Katherine ${ }^{l *}$, González Yurisbeth ${ }^{l}$, Montezuma Génesis $^{l}$, Samudio María ${ }^{l}$ \\ ${ }^{1}$ Licenciatura en Ingeniería Industrial. Universidad Tecnológica de Panamá
}

\begin{abstract}
Resumen Un plato desechable de plástico puede demorar entre 100 y 1000 años para desintegrarse, una bolsa plástica 150 años y las botellas PET demoran hasta 1000 años para degradarse. A pesar de esto, cada día se incrementa el uso de productos plásticos. Por este motivo, se tiene el objetivo de elaborar un material que pueda funcionar para fabricar artículos biodegradables (platos, vasos, cubiertos, bolsas, entre otros), que se puedan desintegrar en menor tiempo, y así ofrecer una solución factible a esta problemática. Para la confección de este proyecto, se investigaron las propiedades de la fibra de coco y la cáscara de plátano para elaborar a partir de cada uno de ellos, junto con otros componentes, el prototipo de material biodegradable. Además, se realizaron pruebas con cada material, controlando la temperatura y las porciones de la materia prima en cada prueba hasta encontrar las cantidades óptimas a utilizar. Por último, se midió el tiempo de desintegración del material al dejarlo a la intemperie, es decir bajo el sol y la lluvia.

El resultado final es un material biodegradable empleando dos materias primas distintas, pero que al comparar su tiempo de degradación es muy similar estando en un rango de 35 a 40 días, lo cual es mucho menor al tiempo que se tenía estipulado (3 meses). Con esto queda comprobado que la fibra de coco y las cáscaras de plátano son una excelente alternativa para reemplazar en un futuro cercano el tan contaminante plástico.
\end{abstract}

Palabras clave Materiales biodegradables, fibra de coco, cáscara de plátano, bioplástico, plástico.

\begin{abstract}
A disposable plastic plate can take between 100 and 1000 years to disintegrate, a plastic bag 150 years and PET bottles take up to 1000 years to degrade. Despite this, the use of plastic products is increasing every day. For this reason, the objective is to develop a material that can work to manufacture biodegradable items (plates, glasses, cutlery, bags, among others), which can disintegrate in less time, and thus offer a feasible solution to this problem. For the preparation of this project, the properties of coconut fiber and plantain banana peel were investigated to make the prototype of biodegradable material from each of them, together with other components. In addition, tests were carried out with each material, controlling the temperature and portions of the raw material in each test until the optimal quantities to be used were found. Finally, the decay time of the material was measured by leaving it outdoors, that is, under the sun and rain. The final result is a biodegradable material using two different raw materials, but when comparing its degradation time, it is very similar being in a range of 35 to 40 days, which is much less than the stipulated time (3 months). With this it is proven that coconut fiber and banana peels are an excellent alternative to replace soon the highly polluting plastic.
\end{abstract}

Keywords Biodegradable materials, coconut fiber, banana peel, bioplastic, plastic.

* Corresponding author: katherinecubilla80@gmail.com

\section{Introducción}

El planeta y la humanidad en general enfrentan un grave problema medioambiental que probablemente estará presente por muchos años más, la contaminación por el alto grado de consumo de productos plásticos se apodera cada vez más de nuestros ríos y mares, afectando la vida que hay en ellos y que también nos afecta directamente a nosotros, ya que dentro de nuestra cadena alimenticia se encuentran los productos del mar, los cuales día a día están consumiendo grandes cantidades de micro partículas de plástico. 
En la actualidad se estima que se genera anualmente 1.900 millones de toneladas de residuos sólidos, y casi el 30\% de la misma permanece sin recoger. En cuanto a los residuos sólidos urbanos (RSU) recolectados, el 70\% se dirige a vertederos, el $19 \%$ se recicla o recupera y el $11 \%$ se destina a instalaciones de recuperación de energía [1].

Considerando esto, surge la pregunta, ¿Cómo podemos ayudar al planeta y ayudarnos a nosotros mismos? La solución podría estar más cerca de lo que pensamos; en la propia naturaleza. A través de esta investigación se demuestra como la fibra de coco y la cáscara de plátano pueden ser utilizadas para elaborar un material que sustituya al plástico, y que pueda ser empleado en productos de uso común, como por ejemplo platos y vasos desechables.

El objetivo de este proyecto es desarrollar un material biodegradable que pueda ser utilizado para reemplazar el plástico utilizado en utensilios como vasos, platos, cucharas, etc., y que además al desecharse pueda desintegrarse en un corto tiempo.

Con el uso de materiales biodegradables que reemplacen el plástico, disminuirá la contaminación ambiental, logrando una mayor calidad de vida para el ambiente y los seres humanos.

Con este experimento se busca comprobar que el tiempo de degradación de un material biodegradable elaborado a partir de fibra de coco y cáscara de plátano como materia prima, es igual o menor a tres meses.

\section{Antecedentes}

Uno de los principales problemas a los cuales se enfrenta la humanidad en la actualidad es la contaminación ambiental. Muchas son las fuentes que provocan dicha problemática, sin embargo, la producción desmedida de todo tipo de productos de plástico es la mayor preocupación no solo de esta sino de futuras generaciones. Se toman en consideración aquellos productos que se denominan desechables y que en su mayoría solo se utilizan una vez, pues no hay forma de volverlos a reutilizar. No debemos olvidar otro tipo de producto desechable, aquellos a base de poliestireno, su problema radica en que no son reciclables y mucho menos degradables, y una vez más todos estos desechos llegan al mar donde al fragmentarse en pedazos más pequeños son ingeridos por la fauna marina (peces, tortugas, etc.), esto quiere decir que indirectamente lo que consumimos es plástico.

Como consecuencia de una mala gestión de los residuos o de su abandono, unos 8 millones de toneladas de plásticos acaban en los mares y océanos anualmente, formando el 60$80 \%$ de la basura marina, en su mayoría en forma de microplásticos (fragmentos inferiores a $5 \mathrm{~mm}$ ). Se desconoce la cantidad exacta de plásticos en los mares, pero se estiman unos 5-50 billones fragmentos de plástico, sin incluir los trozos que hay en el fondo marino o en las playas [2]. Tan solo en la ciudad de Panamá, el 30\% de la basura que se produce a diario, es decir, unas 700 toneladas, van a parar al mar, según el Programa de las Naciones Unidas para el Medio Ambiente (Pnuma) y el Ministerio de Ambiente. La mayor parte de esas toneladas de desechos corresponde a plástico [3].

Para mitigar el excesivo uso de este material contaminante, en la Universidad de Chapingo, Texcoco, Estado de México, un grupo de estudiantes desarrolló la idea de crear platos desechables biodegradables empleando fibra de coco, dicho material puede desintegrarse en un lapso de 2 días a 3 meses [4]. A partir de esto la idea que se plantea es el uso de materiales biodegradables para la confección de platos, vasos, cubiertos, y otros productos desechables no solo con fibra de coco sino, aprovechar muchos más productos que normalmente son desechados por no tener el conocimiento de cómo sacarles más provecho, este es el caso de las cáscaras de plátano.

Es importante mencionar, en lo que se refiere al plátano, según datos del Ministerio de Desarrollo Agropecuario (MIDA), el año pasado su producción se incrementó de 268 a 319 cientos por hectárea. Además, la mayor producción se da en la provincia de Chiriquí con un 58\%, 18\% Darién, 15\% Bocas del Toro y el resto 9\% Colón, Herrera, Los Santos, Panamá Oeste y Coclé. Debido a esto, surgió la idea que si se recolecta las cáscaras de estos productos se estaría dando el máximo aprovechamiento a los mismos.

Por otra parte, para la elaboración de un utensilio biodegradable de manera artesanal es importante considerar la resistencia de este a los alimentos, ya sea secos o húmedos, por lo que se propone elaborar una mezcla que le dé resistencia a la superficie del plato la cual es elaborada con ingredientes de uso casero y que no representan ningún peligro para la salud. Debido a que la materia prima utilizada da un color característico al material elaborado, se sugiere la utilización de colorantes vegetales que permitan diversificar el color y apariencia del producto final.

\section{Metodología}

El método utilizado para desarrollar el prototipo de material biodegradable, a partir de fibra de coco y cáscara de plátano, es el científico, ya que se basa en la observación y experimentación. Además, este proyecto tiene un enfoque cuantitativo, porque usa la recolección de datos para probar hipótesis, con base en la medición numérica y el análisis estadístico.

Dicha hipótesis es, que si generamos nuevas alternativas para la elaboración de productos biodegradables (platos, vasos, cubiertos, bolsas, entre otros) a partir de fibras naturales como las del coco o la del plátano, se obtendría un material que al ser desechado se desintegre en un corto plazo. De esta manera 
contribuimos a disminuir la contaminación del planeta, principalmente de los océanos.

Es importante mencionar que se va a controlar el tiempo de degradación del material, para realizar un diseño de experimento, que nos muestre si hay diferencia en la descomposición de los materiales.

En esta investigación se aplica un diseño de bloque completo al azar (DBCA), que consiste en una prueba basada en el análisis de varianza, en el cual se buscan las sumas de los cuadrados, ya sea del tratamiento ecuación 1, suma del cuadrado del error ecuación 3, y del total ecuación 4. También, se incorpora un factor adicional de variabilidad conocido como bloque, donde se obtiene la suma de cuadrado como se muestra en la ecuación 2. Este elemento se incorpora al experimento para determinar si el mismo incide en el resultado de la evaluación del tratamiento. En la ecuación 5 se muestra que si ese parámetro se cumple, las hipótesis no son iguales, ya que si la F de la prueba es mayor a la F crítica se rechaza la hipótesis nula. En caso de que eso ocurra se debe volver hacer el ANOVA pero, solo al tratamiento o bloque que rechazó la hipótesis nula. Y luego se utiliza el método LSD ecuación 6 para la comparación de las medias [5].

$$
\begin{gathered}
s_{c} \text { tratamient } o=\sum_{i=1}^{k} \frac{y_{i}^{2}}{b}-\frac{y_{. .}^{2}}{N} \\
s_{c} \text { bloque }=\sum_{i=1}^{b} \frac{y_{j}^{2}}{k}-\frac{y_{. .}^{2}}{N} \\
s_{c} \text { error }=s_{c} \text { total }-s_{c} \text { tratamient } o-s_{c} \text { bloque } \\
s_{c} \text { total }=\sum_{i=1}^{k} \sum_{j=1}^{n_{i}} y_{i j}^{2}-\frac{y_{. .}^{2}}{N} \\
F_{p}>F_{c} \\
\mathrm{LSD}=\mathrm{t}_{\frac{\infty}{2}}, \mathrm{~N}-\mathrm{k}_{\frac{2 \mathrm{CME}}{\mathrm{n}_{\mathrm{i}}}}
\end{gathered}
$$

Para desarrollar el Diseño de Bloque Completo al Azar (DBCA) se van a recolectar los datos de interés (días en que se desintegra el material), luego introducirlos en Excel, y a través de este se obtiene un análisis de varianza (ANOVA), que se considerada como la técnica central en el análisis de datos experimentales, para luego conocer si existen diferencias significativas entre los materiales y sus respectivos tratamientos.

\subsection{Materiales y Procedimientos}

Los materiales utilizados para la elaboración de material biodegradable a partir de fibra de coco y cáscara de plátano son los que se muestran en la figura 1.

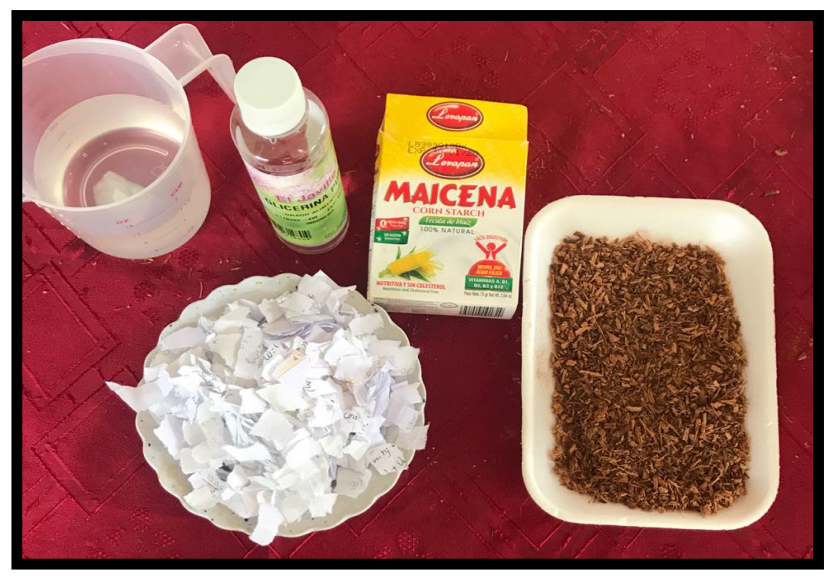

Figura 1. Materiales utilizados.

Materiales Utilizados:

1. Fibra de Coco

2. Cáscara de plátano

3. Papel reciclado

4. Agua

5. Fécula de maíz

6. Glicerina - Grado alimenticio

7. Colorante vegetal

\subsubsection{Propiedades de los materiales Cáscara de plátano}

El principal subproducto del plátano es la cáscara la cual representa aproximadamente el $30 \%$ del peso del fruto; las aplicaciones potenciales para la cáscara de plátano dependen de su composición química. Igualmente es rica en fibra dietética, proteínas, aminoácidos esenciales, ácidos grasos polinsaturados y potasio; entre los esfuerzos para utilizar la cáscara se han obtenido proteínas, metanol, etanol, pectinas y enzimas [6].

\section{Fibra de coco}

La fibra de coco es un producto orgánico y biodegradable con las siguientes características [7]:

- Resistencia

- Durabilidad

- Acción antibacterial

- Gran capacidad de aislamiento térmico y acústico.

A continuación, en la figura 2 se muestra el procedimiento empleado para la elaboración de un prototipo de plato biodegradable [8]. 


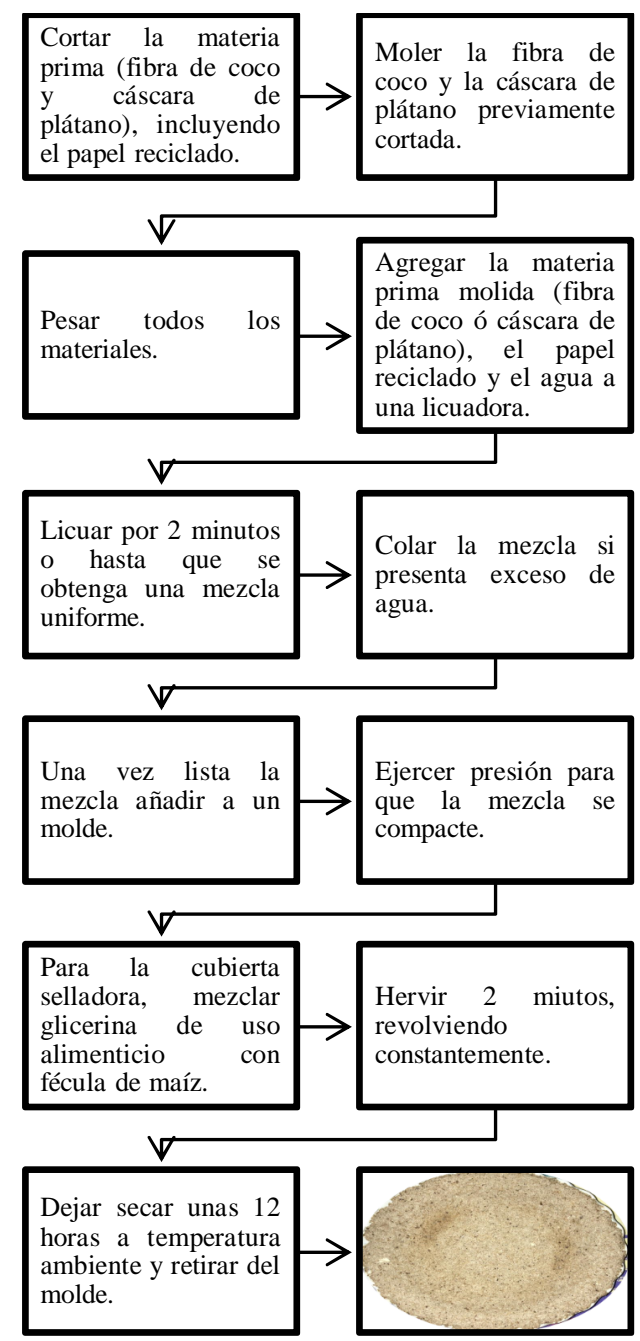

Figura 2. Procedimiento para la elaboración de un prototipo de plato biodegradable.

\section{Resultados}

En la figura 3 se puede observar la primera prueba realizada con un prototipo normal, donde se muestra el material obtenido sin la capa de bioplástico y en la figura 4 los últimos prototipos realizados.

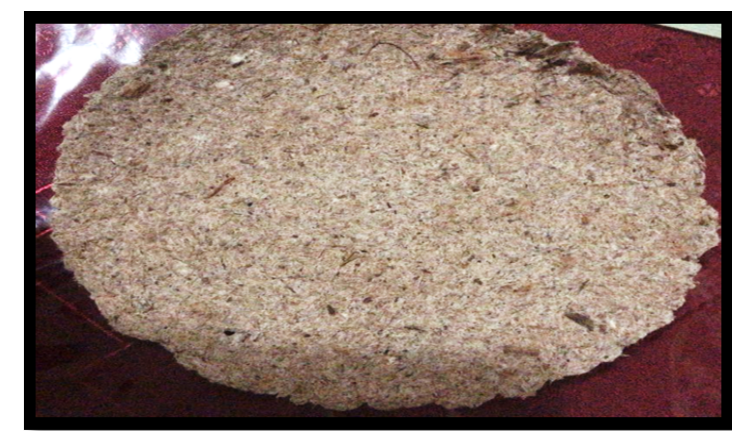

Figura 3. Prototipo normal.

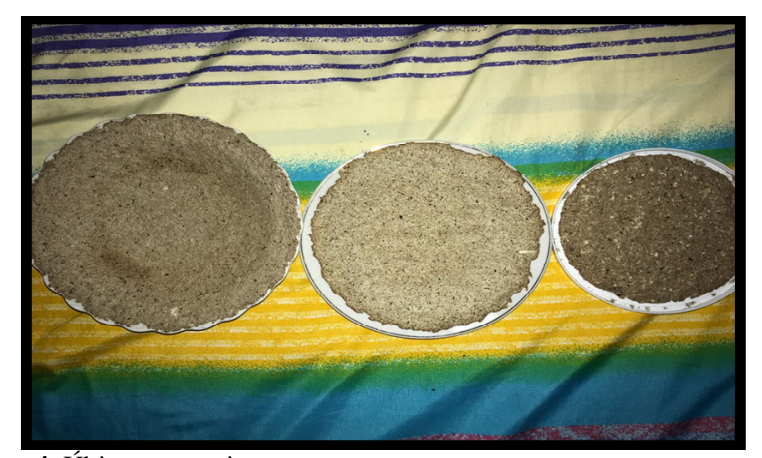

Figura 4. Últimos prototipos.

Luego de hacer las pruebas y obtener los datos, estos se analizaron a través de un diseño experimental (DBCA), donde se busca observar si existe diferencia en el tiempo de degradación de los distintos materiales elaborados.

Se van a realizar distintas pruebas con dos tipos de fibras utilizados en la elaboración de un material biodegradable: fibra de coco y cáscara de plátano, que aparecen en la tabla 1 como MC, MP respectivamente. Se desea comparar el tiempo de degradación de ambos (en días), para saber cuál de ellos se degrada en menor tiempo, y de esta manera sea más beneficioso para el medio ambiente. Para ello, se dejó a la intemperie (bajo la lluvia y el sol), hasta que volviera nada. Se escogieron cuatro prototipos de cada material.

Tabla 1. Datos para el DBCA
\begin{tabular}{|c|c|c|c|c|c|}
\hline & T1 & T2 & T3 & T4 \\
\hline MC & 40 & 38 & 35 & & 40 \\
\hline MP & 31 & 35 & 30 & & 32 \\
\hline
\end{tabular}

Ahora se plantea el problema donde las hipótesis quedan de la siguiente manera:

$$
\begin{aligned}
& \mu_{0}=\mu_{1} \\
& \mu_{0} \neq \mu_{1}
\end{aligned}
$$

La $\mu_{0}$, indica la media del tiempo de desintegración del material biodegradable de coco, mientras que la $\mu_{1}$ indica la media del tiempo de desintegración de material biodegradable de plátano. La hipótesis nula considera que los tiempos de degradación de cada material son iguales, y para la alternativa son distintos.

Tabla 2. Análisis del problema

\begin{tabular}{|c|l|l|l|l|}
\hline RESUMEN & Cuenta & Suma & Promedio & Varianza \\
\hline MC & 4 & 153 & 38.25 & 5.58 \\
\hline MP & 4 & 128 & 32 & 4.67 \\
\hline Tiempo1 & 2 & 71 & 35.5 & 40.5 \\
\hline Tiempo2 & 2 & 73 & 36.5 & 4.5 \\
\hline Tiempo3 & 2 & 65 & 32.5 & 12.5 \\
\hline Tiempo4 & 2 & 72 & 36 & 32 \\
\hline
\end{tabular}


En la tabla 2 se muestran los resultados obtenidos al analizar los distintos tiempos. La tabla 3 muestra la parte final de los datos analizados, donde se puede observar las sumas de cuadrados de los materiales, del tiempo de degradación, entre otros. También se obtienen los grados de libertad, el promedio de los cuadrados (CM) y la F de la prueba Fp y la F critica.

Tabla 3. Análisis de Varianza

\begin{tabular}{|c|r|r|r|r|c|}
\hline \multicolumn{7}{|c|}{ ANÁLISIS DE VARIANZA } \\
\hline $\begin{array}{c}\text { Origen de las } \\
\text { variaciones }\end{array}$ & SC & GL & CM & F $_{\mathbf{p}}$ & $\mathbf{F}_{\mathbf{c}}$ \\
\hline materiales & 78.1 & 1 & 78.1 & 20.6 & 10.1 \\
\hline Tiempo & 19.4 & 3 & 6.5 & 1.7 & 9.3 \\
\hline Error & 11.4 & 3 & 3.8 & & \\
\hline Total & 108.9 & 7 & & & \\
\hline
\end{tabular}

Con los resultados obtenidos podemos concluir que la hipótesis nula se rechaza para el caso de los materiales (fibra de coco y cáscara de plátano). Esto quiere decir que se tiene que volver hacer el ANOVA, pero solo con el tratamiento como se muestra en la tabla 4 , ya que ese fue el que dio diferencia significativa.

Tabla 4. Nuevo ANOVA

\begin{tabular}{|c|c|c|l|l|l|}
\hline \multicolumn{7}{|c|}{ ANÁLISIS DE VARIANZA } \\
\hline $\begin{array}{c}\text { Origen de las } \\
\text { variaciones }\end{array}$ & SC & GL & CM & F & $\mathbf{F}_{\mathbf{c}}$ \\
\hline Materiales & 78.1 & 1 & 78.1 & 15.2 & 6.0 \\
\hline Error & 30.8 & 6 & 5.1 & & \\
\hline Total & 108.9 & 7 & & & \\
\hline
\end{tabular}

Luego que se hace el nuevo ANOVA se procede a utilizar el método de diferencia mínima significativa (LSD), como se muestra en la tabla 5. El método de diferencia mínima significativa (LSD) se refiere a la diferencia mínima que debe haber entre dos medias muestrales, para considerar que dos tratamientos son diferentes.

Tabla 5. Resultados del LSD

\begin{tabular}{|l|l|c|c|c|}
\hline \multicolumn{2}{|c|}{ LSD } & 3.92 & & \\
\hline \multicolumn{2}{|l|}{$\mid \mathbf{y 1 - y 2}$} & 6.25 & $>$ & 3.92 \\
\hline
\end{tabular}

En los resultados obtenidos que el valor de LSD es menor con respecto al otro. Lo que nos lleva a concluir que, si hay diferencia significativa, y que el material con menor tiempo de degradación es el de cáscara de plátano.

Luego de este análisis se muestra la tabla 6, con la evidencia del material que se puso a descomponer.
Tabla 6. Descomposición del material

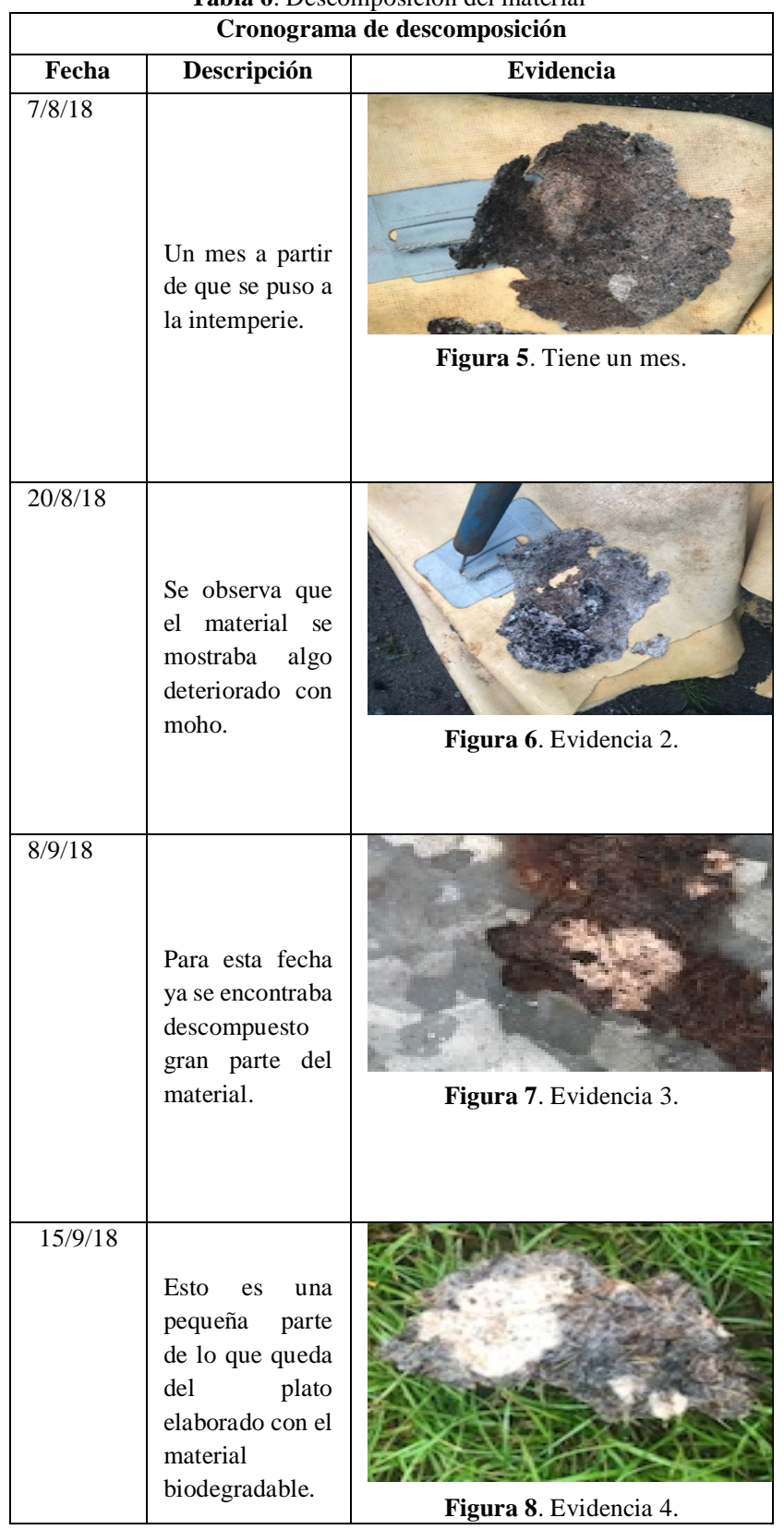

El material biodegradable obtenido fue sometido a pruebas de tipo organolépticas, para descartar olores desagradables, con esto se comprobó que el material presentaba un olor poco perceptible, a través del tacto se verificó la textura del material la cual es rugosa debido al tipo de materia prima que se empleó. Para las pruebas físicas se comprobó la degradación del material en pocos meses, su resistencia a los líquidos al colocarle alimentos húmedos por un período de tiempo de cinco minutos y su resistencia a la tracción, al estirarlo y doblarlo. 


\section{Conclusiones}

Los desechos sólidos cada día están deteriorando nuestro planeta y debemos buscar alternativas eco-amigables que nos ayuden a mejorar nuestra calidad de vida. Según lo investigado, los plásticos tienen un gran porcentaje en esto y por tal motivo se ha diseñado un material biodegradable, que demore poco tiempo en desintegrase. Las fibras escogidas para esto fueron las del coco y plátano, ya que aquí en nuestro país se producen en gran cantidad.

Con la elaboración de materiales biodegradables es posible disminuir el alto grado de contaminación que existe en la actualidad, tras varias pruebas realizadas se logró obtener un prototipo acorde a nuestros requerimientos empleando materiales $100 \%$ biodegradables manteniendo una buena calidad, textura y resistencia del producto final. Este producto brinda la confianza de un material seguro que después de su uso, su vida útil no será mayor a tres meses.

Los datos obtenidos en el análisis del experimento a través de la herramienta Excel, muestran que el material que tiende a degradarse en menos tiempo es el de cáscara de plátano. Una de las cosas que pueden influir en este resultado son las propiedades funcionales de cada material; ya que la fibra de coco tiene más resistencia y durabilidad.

\section{Recomendación}

Para la elaboración de materiales de este tipo, se pueden utilizar otras materias primas; una alternativa sería la caña de azúcar. Se hizo una prueba con este material como se muestra en la figura 9, donde se obtuvo una textura similar a la del material elaborado con las dos fibras principales de nuestra investigación.

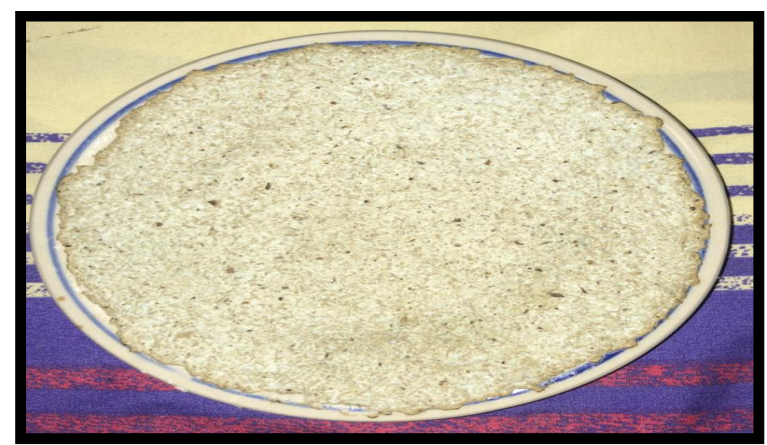

Figura 9. Material a base de caña de azúcar.

Para estudios futuros se puede medir la resistencia al calor, al peso y probar si ese material puede tener otro color con ayuda de colorantes naturales.

\section{REFERENCIAS}

[1] "Waste Atlas," 2013. http://www.atlas.d-waste.com/. [Accessed 10 junio 2018].

[2] "gob mx," $13 \quad$ Octubre 2016. https://www.gob.mx/conafor/prensa/crean-platosdesechables-biodegradables-con-fibra-de-coco. [Accessed 12 Junio 2018].

[3] greenpeace. https://archivoes.greenpeace.org/espana/Global/espana/2016/report/plastico s/plasticos_en_los_oceanos_LR.pdf. [Accessed 11 Junio 2018].

[4] L. Prensa, "Prensa.com," https://www.prensa.com/sociedad/Plastico-marmesa_0_4541545832.html. [Accessed 12 Junio 2018].

[5] H. Gutiérrez, Análisis y Diseño de Experimentos, McGraw Hill .

[6] C. H. I. Arrivillaga, "Síntesis y caracterización de bioplástico a partir de almidón de banano verde," 2013. file:///c:/users/samsung/downloads/s\%c3\%8dntesis\%20y\%20 caracterizaci\%c3\%93n\%20de\%20biopl\%c3\%81stico $\% 20 \mathrm{a} \%$ 20partir\%20de\%20almid\%c3\%93n\%20de\%20banano.pdf.

[7] "ECOLFIBRAS," [Online]. Available: https://ecolfibras.co/fibra-de-coco/.

[8] Juan G., Diego G., "Empaques biodegradables a partir de fibra de plátano para los productos agrícolas de caldas," [Online]. Available:

file://C:/Users/samsung/Downloads/empaque\%20biodegrad able\%20a\%20partir\%20de\%20fibra\%20de\%20platano.pdf.

[9] T. Mario, Estadística, México : PEARSON EDUCACIÓN , 2009.

[10] "Análisis experimental de la elaboración de bioplástico a partir de la cáscara de plátano," [online]. available: file:///c:/users/samsung/downloads/pyt_informe_final_proyec to_bioplastico.pdf.

[11] Gabriela B., Francisco G., "Propiedades fundamentales del plátano," $2014 . \quad$ [Online]. Available: https://www.uv.mx/rm/num_anteriores/revmedica_vol14_nu $\mathrm{m} 2 /$ articulos/propiedades.pdf. 